\title{
Ecología y Monitoreo del Lagarto Americano para el Plan Comprensivo de la Restauración de los Everglades 1
}

Ken G. Rice, Frank J. Mazzotti y Marianna D.Conill ${ }^{2}$

\section{Introducción}

El lagarto Americano una vez ocupó todos los humedales de la Florida del Sur, desde cenotes y pozos en montes de coníferas, hasta pantanos de agua dulce y estuarios de manglar. Casi todos los seres acuáticos en los Everglades se ven afectados por los lagartos (Beard, 1938). Como predadores superiores en su ecosistema, sufren un extraordinario cambio en cuanto a tamaño corporal y consumen distintos organismos presa durante su crecimiento. Como ingenieros del ecosistema, los senderos y huecos construidos por los lagartos proveen refugio a vadeadores y peces durante la época de sequía, y sus nidos proveen áreas elevadas para otros reptiles y para la germinación de plantas que son menos tolerantes a las inundaciones.

Las prácticas de manejo de aguas y de desarrollo en la Florida del Sur han reducido la

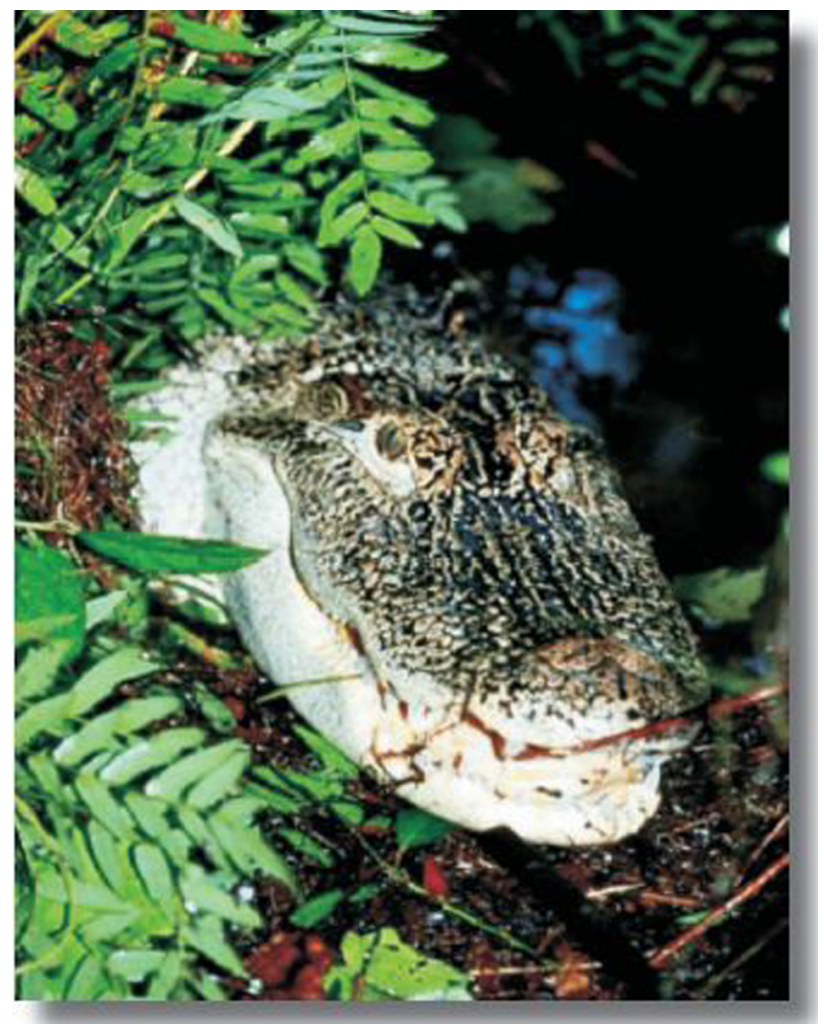
cantidad y calidad de estos hábitats. Menor cantidad

\footnotetext{
1. Este documento es Cir 1478S, perteneciente a la serie del Departamento de Ecología y Conservación de Fauna Silvestre, University of Florida, Cooperative Extension Service, Institute of Food and Agricultural Sciences. Fecha de publicación: Noviembre, 2005. Visite la página web http://edis.ifas.ufl.edu. Imprímase a color para mejor comprensión de gráficos y figuras.

2. Ken G. Rice, U.S. Geological Survey, 3205 College Ave, Davie, FL 33314, e-mail: ken_g_rice@usgs.gov. Frank J. Mazzotti, University of Florida, 3205 College Ave, Davie, FL 33314, e-mail: fjma@ufl.edu. Marianna Domínguez Conill, University of Florida, 3205 College Ave, Davie, FL 33314, e-mail: mconill@ufl.edu.
}

El Instituto de Alimentos y Ciencias Agrícolas es un empleador que opera bajo Acción Afirmativa y provee Oportunidades Igualitarias, autorizado a proveer investigación, información educativa y otros servicios, únicamente a los individuos e instituciones que operan sin discriminación alguna con relación al credo, color, religión, edad, incapacidad, sexo, orientación sexual, estado civil, nacionalidad, opinion política o afiliaciones. Para más información sobre como obtener otras publicaciones de extensión, comuníquese con la oficina de Servicio de Extensión de su condado. Servicio de Extensión de la Florida / Instituto de Alimentos y Ciencias Agrícolas / Universidad de la Florida / Larry Arrington, Decano. 
de agua dulce fluye hacia las zonas de manglares, lo que resulta en una mayor salinidad y en la sedimentación de riachuelos (Craighead, 1971). En praderas de marga, los históricos hidroperíodos de 6 a 9 meses de duración, han sido reducidos a 3 meses y en ocasiones hasta menos. Adicionalmente, los niveles de agua pueden disminuir hasta $60 \mathrm{~cm}$ por debajo de la superficie de la tierra durante la época de sequía. Como consecuencia de estos cambios, hoy en día los lagartos son menos numerosos en algunos hábitats (Mazzotti y Brandt, 1994). Como Craighead (1968) declara, "probablemente ningún fenómeno caracteriza mejor la profanación del hombre de la región primitiva del sur de la Florida que la desaparición del lagarto con sus ignorantes tentativas de manipular el plan de la naturaleza de regar los Everglades".

Actualmente, se está comenzando la restauración bajo el Plan Comprensivo de la Restauración de los Everglades (CERP, por sus siglas en inglés; $w w w$.evergladesplan.org), el cual incluye más de 68 proyectos, desde la remoción de canales hasta la creación de áreas de almacenamiento de agua alternas. Los objetivos del plan incluyen el incremento de la extensión espacial de áreas naturales y el mejoramiento tanto de la calidad de las áreas naturales existentes como de la abundancia y diversidad de plantas y animales nativos.

Un componente importante del plan es un proceso de evaluación adaptativa que será utilizado para medir el desempeño de CERP y poder recomendar cambios como fuesen necesarios. Parte del proceso de evaluación adaptativa incluye la identificación de fauna y hábitats claves que puedan servir como indicadores del éxito. El lagarto Americano fue elegido por su relación con la hidrología, salinidad y productividad del sistema, los cuales deben ser afectados durante la restauración.

Muchas preguntas respecto a los efectos de la restauración de los Everglades sobre las poblaciones de lagartos aún permanecen en cuestionamiento, tales como los impactos de la remoción de canales, el rol de los huecos de lagartos como refugios acuáticos y los efectos de la hidrología sobre la condición y el crecimiento de las poblaciones. Además, no se han desarrollado métodos para monitorear y evaluar el éxito de restauración a lo largo del sistema de los Everglades. Adicionalmente, necesitamos continuar actualizando y validando herramientas de restauración tales como modelos poblacionales para el uso en selecciones alternativas, desarrollo de medidas de rendimiento y predicción de los efectos de restauración. Este proyecto, un esfuerzo cooperativo entre el U.S. Geological Survey (USGS) y University of Florida, tratará directamente las preguntas mencionadas anteriormente, desarrollará métodos de monitoreo y validará herramientas de restauración para utilizar en CERP.

\section{Abundancia del Lagarto}

Los cambios en el uso y la cobertura de las tierras como resultado del manejo de aguas han afectado las poblaciones de lagartos. Algunas localidades que fueron hábitat del lagarto han sido convertidas en desarrollos residenciales y agrícolas. Otras localidades están tan drenadas que los lagartos sólo se encuentran en cuerpos de agua permanentes, como canales o pozos, o durante períodos de niveles de agua extremadamente altos. Mientras el objetivo de restauración de los Everglades se enfoque en la restauración de la hidrología en estas áreas, la reaparición de lagartos será un excelente indicador del éxito de restauración.

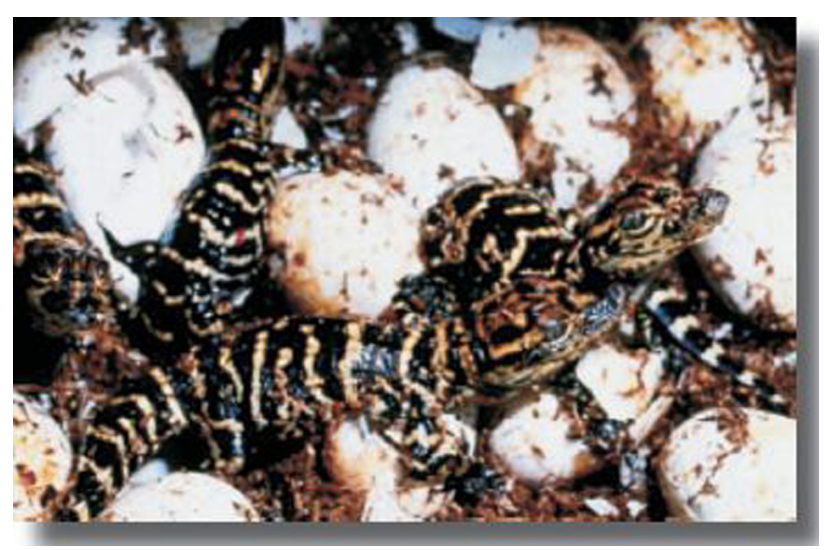

Actualmente, los únicos monitoreos a largo plazo de lagartos en los Everglades son conteos aéreos de nidos. El Florida Fish and Wildlife Conservation Commission conduce limitados estudios nocturnos de lagartos en Water Conservation Areas 2 y 3 (WCA2 y WCA3, respectivamente) y sus 
adyacencias como parte de su proyecto de monitoreo de las poblaciones de lagarto a lo largo del estado, y realiza conteos aéreos de nidos como parte del programa de colección de huevos en aguas públicas. Aún cuando se lleva a cabo la restauración en distintos ecosistemas, tales como las zonas rocosas denominadas "rocky glades" y las zonas de transición del manglar, tomará más de 10 años para que los juveniles en dispersión pasen a ser animales capaces de hacer nidos. De esta manera, evaluar la distribución, abundancia y demografía relativa de lagartos permite un estudio más rápido y geográficamente balanceado de los impactos de los proyectos de CERP en los sistemas objetivo.

Hoy en día se han establecido rutas de estudio en ríos estuarinos, canales de agua dulce y pantanos que se extienden desde el margen de los manglares en el Everglades National Park (ENP) hasta el norte del Loxahatchee National Wildlife Refuge (Fig. 1). Durante estos estudios contamos, estimamos los tamaños, y llevamos un récord de las localidades de lagartos en una extensión de más de 300 kilómetros de rutas de botes de aire, ríos y canales en los Everglades. Estos estudios se llevan a cabo 4 veces al año para estimar tendencias en las poblaciones. Actualmente, estamos iniciando estudios para mejorar nuestras estimaciones del número total de lagartos presentes en las rutas mediante el uso de radiotelemetría y técnicas de estimación estadística. Para evaluar el éxito de restauración, esta red de estudios finalmente podrá detectar cambios en las poblaciones de lagartos en el orden del 5 por ciento anualmente, en un período de 2-3 años. La red de estudio del lagarto descrita anteriormente es el primer esfuerzo sistemático de total cobertura del sistema que monitorea las poblaciones de lagartos de los Everglades.

\section{Condición del Lagarto}

La condición corporal (la relación entre el largo del cuerpo y el peso) de los lagartos puede cambiar dentro de un período corto de tiempo cuando se altera la cantidad de presas disponibles. En los Everglades, esto se encuentra estrechamente relacionado con la hidrología. Cuando aumenta la profundidad del agua, las presas se dispersan en el medio, los lagartos encuentran menos presas y la

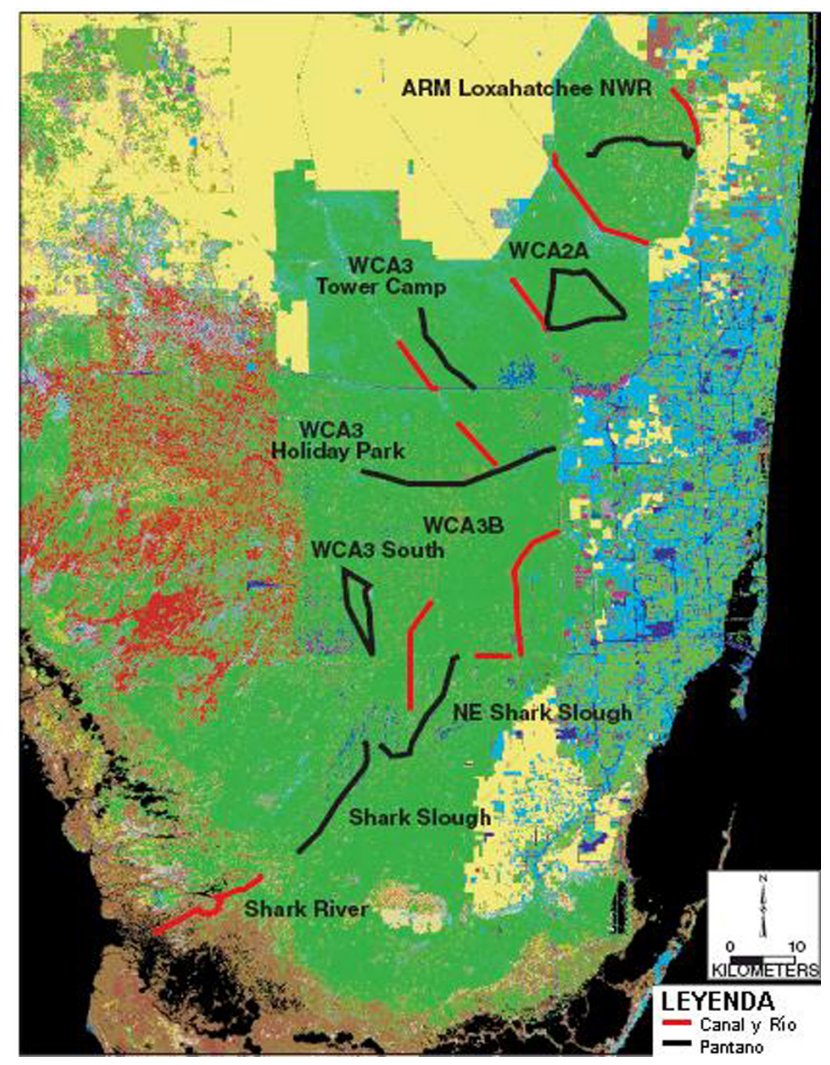

Figura 1. Estudio de las rutas de estudio de lagartos en los Everglades. Credits: Hardin Waddle

condición corporal disminuye. Cuando disminuye la profundidad del agua, las presas se concentran en los huecos de lagartos, los lagartos capturan una mayor cantidad de presas y la condición corporal aumenta. Por supuesto, si la profundidad del agua disminuye demasiado o se mantiene baja por mucho tiempo, el número de presas disminuye y la condición corporal de los lagartos se ve afectada o recurren al canibalismo, o ambas.

La condición corporal ha sido de interés para los investigadores ya que provee una forma de evaluar cuán bien se acoplan los cocodrílidos a los cambios en el medio ambiente. Las medidas de condición corporal pueden proveer una medida del éxito de restauración a través de una evaluación de los lagartos en los Everglades espacial y temporalmente. La condición puede verse como una medida de la accesibilidad y calidad de las especies presa y provee una conexión con los niveles tróficos más bajos y su éxito durante la restauración. El monitoreo de la condición tanto en hábitats recientemente restaurados, como los "rocky glades", y en centros de poblaciones existentes es crítico para el entendimiento de los efectos de la restauración. 
Hemos establecido sitios de monitoreo a lo largo de los Everglades para evaluar la condición corporal y para desarrollar relaciones entre hidrología y condición corporal. Hasta el presente día, hemos capturado más de 1000 lagartos (Fig. 2) para evaluar la condición corporal a lo largo de los Everglades. Durante los primeros 4 años de monitoreo hemos visto una disminución en todas las áreas (Fig. 3). Ésto podría representar sólo un efecto de corto plazo debido a los patrones de lluvias de la Florida del sur, pero aún así demuestra la sensibilidad a cambios hidrológicos del programa de monitoreo y la utilidad de estos datos durante la restauración.

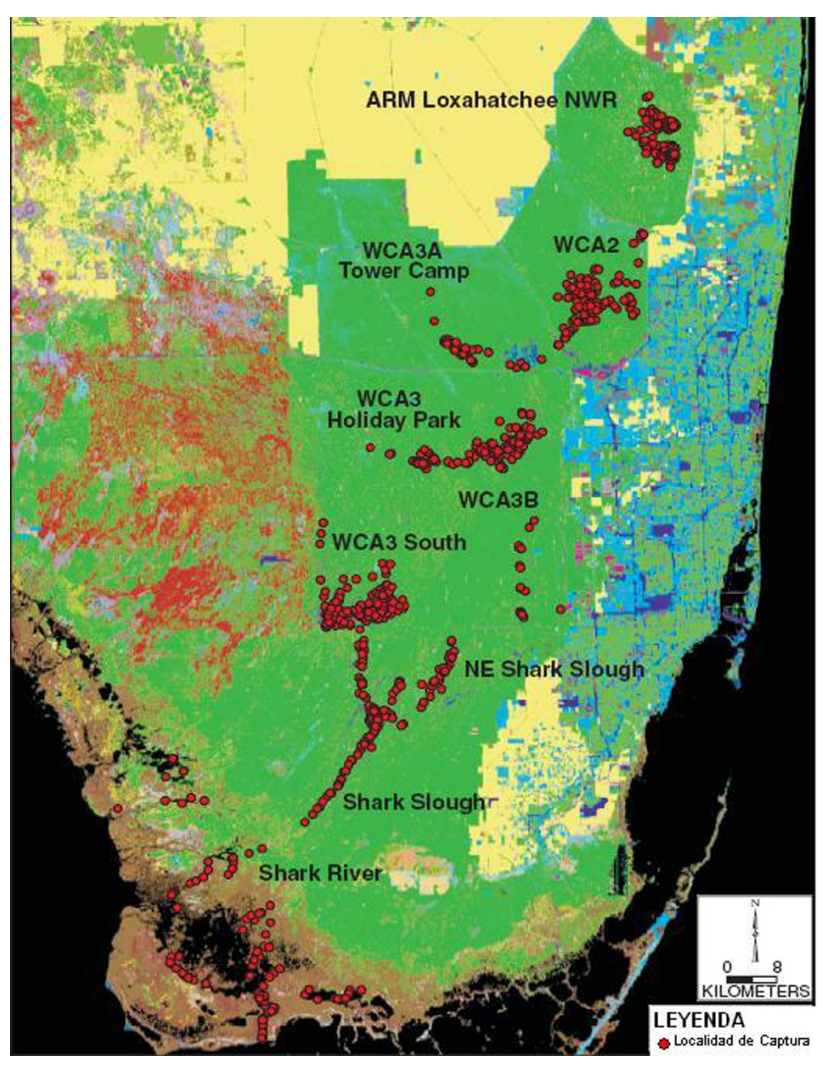

Figura 2. Localidades donde se han capturado lagartos para el estudio de condición corporal en los Everglades.

\section{Huecos de Lagartos}

Los huecos de lagartos son excavados del estiércol húmedo y la turba que conforman los suelos de los Everglades, muchas veces hasta la profundidad de la capa de piedras calizas. Estas depresiones proveen un refugio acuático para los lagartos y para otros organismos acuáticos, así como sitios de anidación para otros reptiles y sitios de colonización para plantas de los Everglades. Los huecos de lagartos también proveen sitios de

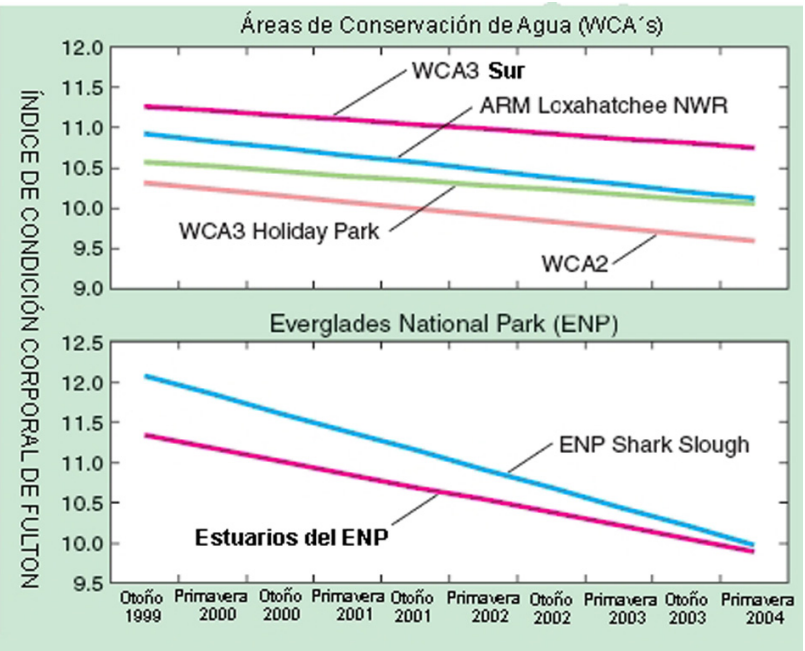

Figura 3. Condición Corporal del lagarto en los Everglades desde 1999 hasta 2004.

forrajeo para vadeadores, tortugas y culebras, y pueden proveer sitios de refugio para peces durante la época de sequía. Los peces que sobreviven la época de sequía en huecos de lagartos conforman una de las fuentes para futuras poblaciones de peces. Además, los senderos de lagartos, libres de vegetación alrededor de los huecos de lagartos, pueden resultar como una franja cortafuego que provee protección para la vegetación leñosa y para varias especies de animales (Craighead, 1968).

Aún cuando los huecos de lagartos y otros refugios de uso durante la época de sequía han sido reconocidos como componentes críticos del ecosistema de los Everglades, hasta recientemente sólamente un hueco de lagarto había sido estudiado en detalle (Kushlan, 1972). Los estudios actuales se han concentrado en el rol ecológico y los patrones de paisaje de los huecos de lagartos en los Everglades. Mientras que estos estudios pueden reiterar la importancia de los huecos de lagartos en los Everglades y proveer enlaces entre las especies necesarias para refinar los modelos ecológicos, el monitoreo de la densidad y ocupación de los huecos de lagartos es esencial para la evaluación del éxito de la restauración.

Actualmente estamos creando mapas con las localidades de los huecos de lagartos a lo largo de los Everglades como base para modelos ecológicos de lagartos, vadeadores, peces y otras especies. Este esfuerzo ha identificado varias relaciones clave que son críticas para la restauración, incluyendo una 


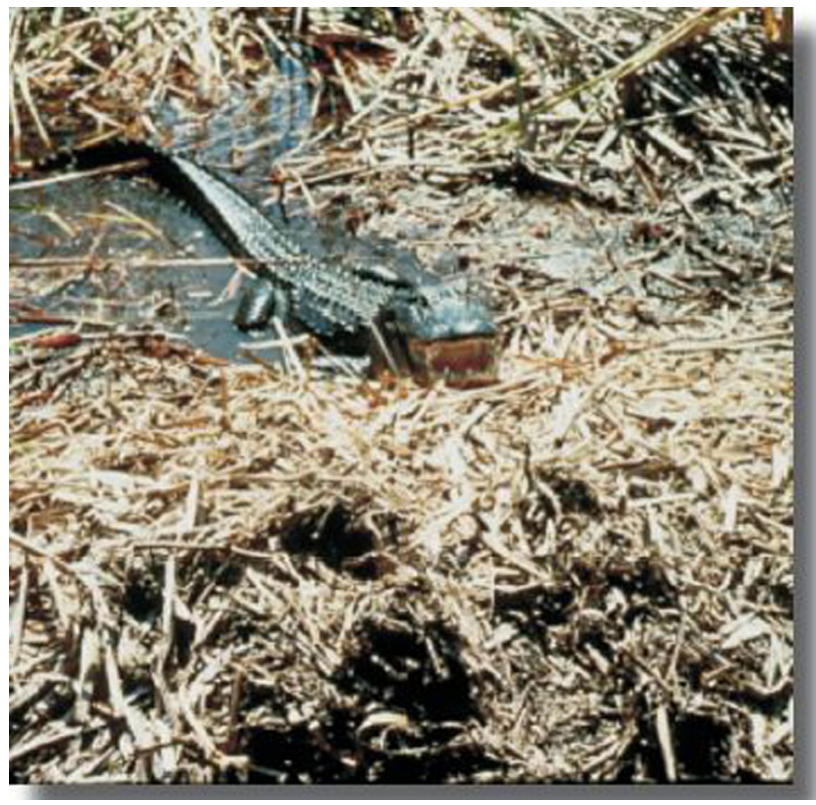

disminución en la densidad de huecos de lagarto cerca de los canales (Fig. 4). Una menor cantidad de huecos de lagarto conlleva a una menor cantidad de refugios y sitios de alimentación para vadeadores, peces y otros reptiles, ya que los canales son muy profundos para el forrajeo de vadeadores. Además, estamos desarrollando un programa de monitoreo para encontrar las tendencias en la densidad y ocupación de los huecos de lagarto. Estamos planificando transectas aéreas a lo largo de los Everglades para evaluar los cambios en cuanto a número, tamaño y tipos de huecos de lagarto y la presencia de lagartos. Un aumento en el número de huecos de lagarto y de lagartos que los mantienen sería un indicador del éxito de restauración en hábitats drenados excesivamente.

\section{Modelos para Lagartos}

La predicción de cómo las alternativas de restauración afectarán las poblaciones de fauna silvestre y los patrones de comunidad es una tarea que el USGS y sus cooperadores han emprendido en el proyecto "Simulación del Sistema a través de los Niveles Tróficos" (“Across Trophic Levels System Simulation" o ATLSS). Este conjunto de modelos de paisaje de las condiciones hidrológicas y las poblaciones de plantas y animales es un modelo a gran escala y espacialmente explícito, que incluye el modelo de población del lagarto Americano (APM, por sus siglas en inglés). Mediante la aplicación del APM a los proyectos de restauración propuestos, a

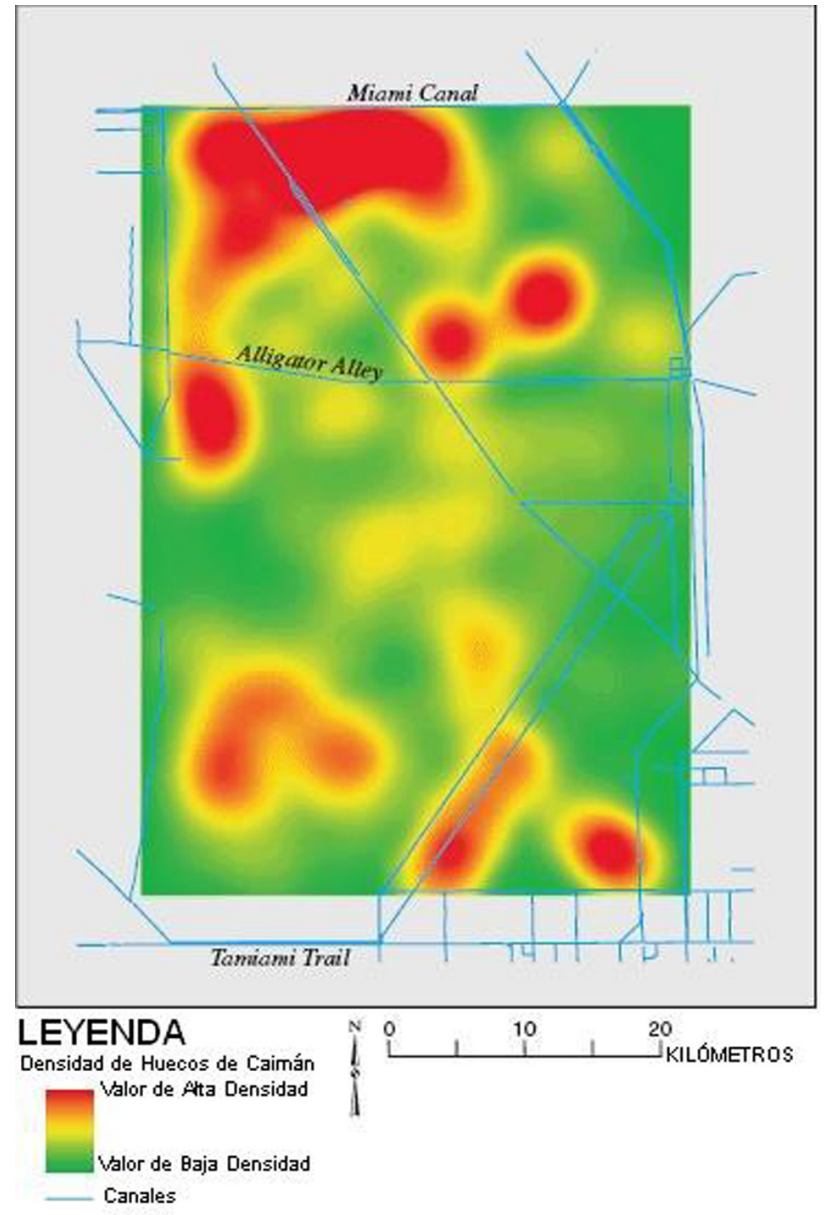

Figura 4. Densidad de huecos de lagarto en el Water Conservation Area 3.

los programas de manejo hidrológico actuales y a las condiciones históricas, podemos examinar y comparar las predicciones de los modelos en cuanto a los efectos sobre la densidad poblacional del lagarto, supervivencia, reproducción y dispersión. Los gerentes de la restauración pueden utilizar los resultados de los modelos para hacer decisiones acerca de cuáles alternativas se deben implementar y monitorear.

Las comparaciones de las condiciones base (1995 y 2050) en la alternativa CERP elegida, D13R, sugieren que las poblaciones de lagartos van a incrementar después de la restauración en muchos de los humedales de los Everglades que han sido drenados excesivamente (Figs. 5 y 6). Sin embargo, el modelo predijo que algunos hábitats, como el Shark River Slough en ENP, el cual se proyecta a tener un aumento en las profundidades de agua bajo CERP, puede que mantengan las poblaciones en los niveles en que se encuentran actualmente o que experimenten 
una leve declinación. Nuestros resultados concuerdan con las predicciones hechas por biólogos especializados en cocodrílidos que trabajan en los Everglades.

Continuaremos actualizando y validando el modelo cada vez que se disponga de nuevos resultados de investigación. Por ejemplo, hemos desarrollado rutas "virtuales" de conteo nocturno con lámparas encandiladoras que se corresponden con nuestras redes de monitoreo para determinar cuán bien el modelo predice las condiciones actuales. El modelo puede ser utilizado para asistir a los gerentes en hacer decisiones informadas hasta que se propongan nuevas alternativas.



Figura 5. Simulaciones del Modelo de Población del Lagarto de CERP contra la Base de 1995.



Figura 6. Simulaciones del Modelo de Población del Lagarto de CERP contra F2050.

\section{Éxito de Restauración}

Durante la restauración de los Everglades, los gerentes requerirán predicciones acerca de cómo los cambios propuestos podrían afectar las poblaciones de especies indicadoras y necesitarán datos de monitoreo que aseguren que se ha logrado restaurar. Se necesitará que esta información abarque la total extensión espacial de los Everglades y períodos cortos y largos de tiempo. Nuestro programa provee un conjunto de herramientas que incluye modelos de predicción (APM) y monitoreo sobre períodos a corto (condición corporal), medio (distribución del lagarto) y largo (hueco de lagarto) plazo, que ocurren a lo largo del sistema de los Everglades. El USGS Priority Ecosystem Science (PES), programa que apoya este estudio, ha sido de gran apoyo en la adquisición de fondos del U.S. Army Corps of Engineers y del Plan de Monitoreo y Evaluación de CERP para continuar esta investigación, incluyendo nuevos componentes de monitoreo, tal como la anidación de lagartos.

\section{Referencias Bibliográficas}

Beard, D.B., 1938, Everglades National Park Project: Wildlife Reconnaissance: U.S. Department of the Interior, National Park Service, Washington D.C.

Craighead, F.C., Sr., 1968, The role of the alligator in shaping plant communities and maintaining wildlife in the Southern Everglades: Florida Naturalist, 41:2-7, p. 69-74.

Craighead, F.C., Sr., 1971, The trees of south Florida: The natural environments and their succession: Miami, Florida, University of Miami Press.

Kushlan, J.A., 1972, An ecological study of an alligator pond in the Big Cypress Swamp of Southern Florida: MS Thesis, University of Miami, Coral Gables, Florida.

Mazzotti, F.J. y Brandt, L.A., 1994, Ecology of the American alligator in a seasonally fluctuating environment; en S.Davis y J. Ogden, (eds.), Everglades: The Ecosystem and its Restoration: Delray Beach, Florida, St. Lucie Press, p. 485-505. 